\title{
Radical Resection HPB Tumors Presenting as Metastatic Lesions: Report of 2 Cases and Literature Review
}

\author{
Monika Gupta* \\ Department of GI, HPB and Minimal Invasive Surgery, India
}

*Corresponding author: Monika Gupta, Department of GI, HPB and Minimal Invasive Surgery, India

Submission: 阱September 09, 2017; Published: 阱 November 09, 2017

\begin{abstract}
Introduction: Pts with resettable tumors may have varied presentations. Few of the tumors can present like advanced tumors with metastasis. These cases should be properly assessed regarding lesions masquerading metastasis with having separate resettable pathology.

Methods: We have successfully managed 2 such cases which presented to us with features suggestive of advanced malignancy. In both of cases hypoprotinemic ascetic with primary localized GB mass and tubercular ascetic with encapsulated cystic pancreatic neoplasm, was masquerading advanced metastatic malignancy respectively. These pts underwent treatment of hypoprotinemic and abdominal tuberculosis respectively, followed by reasonable improvement in general condition as well as disappearance of ascetic and abdominal nodules respectively. Case 1 underwent diagnostic laparoscopy elsewhere followed by, radical cholecystectomy with port site excisions $2.5 \mathrm{yrs}$ back at our centre and later underwent multiple port site hernioplasty, case 2 underwent laparotomy and staging with biopsy and 2 month later she underwent spleen preserving distal pancreatectomy.
\end{abstract}

Result: Both pts underwent 2 operations and they are disease free and surviving till now after 2.5 and 2 yrs respectively.

Conclusion: Proper workup and clinical assessment is necessary before declaring any pancreaticobiliary tumor unrespectable.

Keywords: Metastatic malignancy; Ca gb; Cystic pancreatic neoplasm; Radical resection; Tubercular ascetic; Hypoprotinemic ascitis

Keywords: Resettable HPB tumors; Metastatic presentation; Hypoprotinemic ascitis; Tubercular ascetic; Radical cholecystectomy; Radical distal pancreatectomy

\section{Introduction}

\section{Case 1}

$60 \mathrm{yr}$ old lady referred to department of GI and HPB surgery for management for GB with ascetic, who underwent diagnostic laparoscopy for supposed to be metastatic ca GB at oncology hospital. Presenting features-Pain and distension abdomen, vomiting, generalized edema for 10 days. Diagnostic laparoscopy and biopsy findings of which were-diffuse ascitis with frozen subheptic region with GB not visualized, was biopsy negative for malignancy (exact site not mentioned). 0/E- pt ASA GR3 pedal edema+, ascetic +, abdomen soft no s/o icterus/Lymphadenopathy/peritonitis /Mets Investigations-Hb-9.8gm/dl-, TLC-12700, DLC-N-74\%, L-22\%, E-2\%, M-2\%, urea-17 creatinine-0.6, HIV, HBS Ag, anti HCV -ve, serum bilirubin- 0.3, SGOT-98, SGPT-78, ALP-327, alb -2.3 CXR-B/L pleural effusion, tapping done. USG-CBD normal, mass in GB lumen not involving liver, liver normal, moderate ascitis. Ascetic cytologyve CTSCAN - Mass in GB lumen localized to GB wall filling the lumen with no Mets /LNs, ascetic+ ca 19-9 -3u/ml. Management -She was treated for gastritis, hyponatremia, hypoprotinemia with
PPIs, high protein diet, albumin infusion \&TPN for 7 days. After nutritional build up reevaluation showed serum albumin- 3.1gm/ dl, CECT findings same with resolution of ascitis. The ascitis was a result of hypoprotinemia as ascetic cytology was negative which disappeared after protein replacement. So decision was taken to proceed with diag. lap \&radical cholecystectomy. Intraoperative Findings: Diag. lap-no free fluid, no Mets.

Open: There was localized T2 tumor inside GB lumen no s/o hepatic infiltration, no Mets no HDL/Interaortocaval nodes. Radical ccx with segment $4 b+5$ resection with standard lymphadenectomy and port site excision done (pic1) HPE -gross-polypoid mass GB lumen with a stalk attached to body of GB (pic-2). MicroscopyPapillary adenocarcinoma GB (T2, N0 (0/10 ln), M0), no port site Mets.

\section{Postoperative course}

Pt remained stable oral diet started on day 1, she was discharged on day5. follow up-pt followed up for 2.5yrs every 3 
months and USG done every 3m, CTSCAN, LFT and CA19-9 at 6m show no recurrence/derangements .Pt developed incisional hernia at port excision sites managed with mesh hernioplasty after 2.5yrs after doing repeat restaging by CT SCAN abdomen.

\section{Discussion}

It's known in literature that any mass in GB can metastasize early without causing very big lesion at primary site and may have dismal prognosis. But it's also logical that any mass which is limited to GB wall has high probability of being localized, if clinical picture suggests this ,the case should be reevaluated individually and fair chance should be given for radical excision even in cases of GB malignancies [1]. According to literature ascitis can be differentiated from malignant ascitis by absence thickening of peritoneum, density $6 \mathrm{HU}$ and ascetic cytology being negative [2]. CA19 is increased in 50\%-79\%. CEA \& CA 125 have limited role in conformation. PET CT has no significant role in diagnostic evaluation of Cancer GB over MDCT [3]. Although good results have been reported for cancer GB with single organ involvement but no similar case as ca GB with hypoprotinemic ascetic is reported in literature [4].

\section{Case 2}

Presenting features -A 60 yrs old lady presented with progressively increasing painless lump left side upper abdomen for 2yrs 0/E - no icterus / pallor/edema /Lymphadenopathy. P/A$15 \times 15 \mathrm{~cm}$ non tender mass in left hypochondria.

Investigation: $\mathrm{Hb}-9.1 \mathrm{gm} / \mathrm{dl}$, TLC-4900, DLC-N-86\%, L-10\%, E-2, M-52\%, urea-14, creat-0.6, HIV, HBS Ag, Anti HCV negative, serum bilirubin-0.2, SGOT-18, SGPT-24, ALP-86, alb -2, ca 19-95ng/ $\mathrm{ml}$, Amylase-37, Lipase-28, Serumca 19-95u/ml.

\section{Ascetic fluid Albumin- 1.8, rest-NAD.}

2. USG with doppler SP- Axis was done showed-15x $15 \mathrm{~cm}$ mass near tail of pancreas with fluid, no wall thickening, No involvement of SA SV, No collaterals.

3. Cect- was done showed $15 \mathrm{x} 15 \mathrm{~cm}$ cystic mass in tail of pancreas with setae, no solid areas, No involvement of SA/SV, moderate ascetic + , rt pleural effusion + .

4. Ascetic fluid cytology- -ve.

5. Management- After proper preoperative evaluation pt was taken for laparotomy and spleen preserving distal pancreatectomy.

6. Operative findings-There was $20 \mathrm{x} 20 \mathrm{~cm}$ tense cystic mass in tail of pancreas with multiple nodules in peritoneal cavity with ascetic. Biopsy was taken and abdomen was closed. The differential diagnosis was -either abdominal tuberculosis with CPN or metastatic CPN.

7. HPE of peritoneal nodules- Cascading granulomas, AFB-ve.

8. Postoperative Course- pt was given ATT for 8 months. After 2 months pt. Improved and ascitis resolved. Reassessment done with CECT abdomen showed same findings with resolved ascitis.
9. $2^{\text {nd }}$ surgery was done - Spleen preserving distal pancreatectomy with saving SA and SV both.

10. Intraoperative finding of definitive surgery- No nodules/ ascetic. 20x $20 \mathrm{~cm}$ mass in lesser sac at body and tail of pancreas, medial extent of tumor reaching neck of pancreas with splaying of celiac axis at its upper end. Splenic artery and vein adherent to tumor capsule could be dissected off without compromising radicality. Postoperative day3 drain fluid amylase was 15 , drain removed, pt discharged on $4^{\text {th }}$ POD.

11. Histopathology- $20 \mathrm{x} 20 \mathrm{~cm}$ cystic mass with mutinous lining, no atypical cells seen s/o mucinous cyst adenoma pancreas.

12. Follow up- every 3 months for $1.5 y$ rs with USG being normal, pt has gained $10 \mathrm{~kg}$ wt.

\section{Discussion}

Patient has presented with features of advanced tumorlarge pancreatic mass with ascitis, but on final workup she was diagnosed to have Koch's abdomen. Ascitis was diagnosed to be due to tubercular pathology as carcinoma with metastasis in $\mathrm{CPN}$ is very rare [5,6] her tumor markers were normal [7-11] she underwent spleen preserving distal pancreatectomy. Careful preoperative workup established that she had 2 being pathologies which could be cured. This care is rarest as only one case of isolated pancreatic tuberculosis presenting as cystic pancreatic neoplasm is reported in literature [11], but no case is there of abdominal Koch's co existing with CPN and presenting as metastatic tumor.

\section{Conclusion}

Pts with resettable tumors may have presentation as lesions masquerading metastasis with having separate resectable pathology. In both of our cases these pts were doubted to have unresectable pancreaticobiliary tumors. In case 1 the lady not only resolved ascitis but appeared fit and happier after our intervention She underwent radical cholecystectomy and port site excision, later she developed port site hernias which were also operated 2.5yrs after the $1^{\text {st }}$ surgery. In $2^{\text {nd }}$ case pt was cured of abdominal tuberculosis and pancreatic cystic tumor both due to our careful assessment and surgical intervention. Our approach in this case not only restored our opinion about disease and provided fair disease free survival and also their outlook for life has changed .Once timely and properly managed pts with HPB tumors can survive for long. Careful preoperative assessment could save 2 pts life with reasonable survival benefit.

\section{References}

1. Pezzilli R, Calculli L, Melzi d'Eril G, Barassi A (2016) Serum tumor markers not useful in screening patients with pancreatic mucinous cystic lesions associated with malignant changes. Hepatobiliary and Pancreatic Diseases International 15(5): 553-557.

2. Tsukada K, Takada T, Miyazaki M, Miyakawa S, Nagino M, et al. (2008) Diagnosis of biliary tract and ampullary carcinomas. J Hepatobiliary Pancreat Surg 15(1): 31-40.

3. Debi U, Ravisankar V, Prasad KK, Sinha SK, Sharma AK, et al. (2014) 
Abdominal tuberculosis of the gastrointestinal tract: Revisited. World J Gastroenterol 20(40): 14831-14840.

4. Rissona JR, Macoveia I, Loockb M, Paquettec B, Martina M, et al. (2012) Cirrhotic and malignant ascites: Differential CT diagnosis. Diagnostic and Interventional Imaging 93(5): 365-370.

5. Kalayarasan R, Javed A, Puri AS, Puri SK, Sakhuja P, et al. (2013) A prospective analysis of the preoperative assessment of duodenal involvement in gallbladder cancer. HPB (Oxford) 15(3): 203-209.

6. He XD, Li JJ, Liu W, Qu Q Hong T, et al. (2015) Surgical procedure determination based on tumor-node-metastasis staging of gallbladder cancer. World J Gastroenterol 21(15): 4620-4626.

7. Munekage $M$, Kohsaki $T$, Uemura S, Kitagawa $H$, Namikawa $T$, et al. (2016) Mucinous cystadenocarcinoma of the pancreas with anaplastic carcinoma: A case report and review of the literature. Mol Clin Oncol 4(4): 483-486.
8. Oruç N, Aydın A, Barutcuoğlu B, Aktan C, Nart D, et al. (2015) Cystic fluid chromogranin A levels in different pancreatic cystic lesions. Turk J Gastroenterol 26(6): 522-527.

9. Buscarini E, Pezzilli R, Cannizzaro R, De Angelis C, Gion M, et al. (2014) Italian consensus guidelines for the diagnostic work-up and follow-up of cystic pancreatic neoplasms. Digestive and Liver Disease 46(6): 479493.

10. Falkowski AL, Graber J, Haack HG, Tarr PE, Rasch H, et al. (2013) Isolated pancreatic tuberculosis: A case report and radiological comparison with cystic pancreatic lesions. J Radiology Case Rep 7(1): 1-11.

11. Compagno J, Oertel JE (1978) Mucinous cystic neoplasms of the pancreas with overt and latent malignancy (cystadenocarcinoma and cystadenoma). A clinicopathologic study of 41 cases. Am J Clin Pathol 69(6): 573-580. 